\title{
A Probabilistic Approach to Learn Activities of Daily Living of a Mobility Aid Device User
}

\author{
Mitesh Patel, Jaime Valls Miro and Gamini Dissanayake
}

\begin{abstract}
The problem of inferring human behaviour is naturally complex: people interact with the environment and each other in many different ways, and dealing with the often incomplete and uncertain sensed data by which the actions are perceived only compounds the difficulty of the problem. In this paper, we propose a framework whereby these elaborate behaviours can be naturally simplified by decomposing them into smaller activities, whose temporal dependencies can be more efficiently represented via probabilistic hierarchical learning models. In this regard, patterns of a number of activities typically carried out by users of an ambulatory aid device have been identified with the aid of a Hierarchical Hidden Markov Model (HHMM) framework. By decomposing the complex behaviours into multiple layers of abstraction the approach is shown capable of modelling and learning these tightly coupled humanmachine interactions. The inference accuracy of the proposed model is proven to compare favourably against more traditional discriminative models, as well as other compatible generative strategies to provide a complete picture that highlights the benefits of the proposed approach, and opens the door to more intelligent assistance with a robotic mobility aid.
\end{abstract}

\section{INTRODUCTION \& MOTIVATION}

Automated assistive devices like guiding canes [1], robotic wheelchairs [2], walkers [3] [4] and manipulators [5] are all designed to make life of elderly and disabled people more comfortable by helping them in their everyday activities, enabling them to live more independently and ultimately improving their overall quality of life. With a marked demographic shift at a global level indicating that the worldwide proportion of people aged 60 and over is expected to double between 2000 and 2050 [6], providing the elderly and frail with assistive devices that can enhance their mobility needs in particular has been a strong motivation for researchers to develop more advanced technological aids through alternative or augmentative aid devices [7].

The concept of independence living specifically for the disabled population has been further promoted by the United Nations under the banner of 'Convention on the Rights of Persons with Disabilities (CRPD)' which states that supporting services provided to individuals with a disability should be such that it enables them to be integrated within the community, and not in a segregated settings [8].

With growing advances in low cost sensing devices and computing platforms, it is becoming increasingly viable to advance the development of more intelligent assistive devices able to apply practical aid responsive to the needs of humans

M. Patel, J. V. Miro and G. Dissanayake are with Faculty of Engineering and IT, University of Technology Sydney (UTS), NSW 2007, Australia. (mitesh.k.patelestudent.uts.edu.au, (jaime.vallsmiro, gamini.dissanayake) duts.edu.au) as a tightly knit human-robot interactive system. However, a fundamental challenge in closing the gap between human interactions with machines is the inadequate capability of the devices to become aware of the complex behaviours performed by humans, which is exacerbated by the inherent limitations in the physical sensors used [9]. There has been growing interest in expressing complex behaviours as a combination of meaningful sub-parts referred to as Action Primitives (APs) [10]. Research done on human motion and other biological movements postulates that movement behaviour consists of simple APs, atomic movements that can be combined and sequenced to form complex behaviours [11]. For example, the task of going from a bedroom to the kitchen using a walker can be further decomposed (in reference to a typical house plan, shown as a 2D floor plan in Fig. 2(b)) into: "recall walker $\Rightarrow$ stand up $\Rightarrow$ get out of bedroom $\Rightarrow$ go straight $\Rightarrow$ turn right $\Rightarrow$ go straight $\Rightarrow$ enter kitchen $\Rightarrow$ sit down $\Rightarrow$ indicate walker to go away". Arguments raised in the field of neuroscience [12] reinforces the idea that human actions are composed of APs similar to human speech, where utterances of words are broken down into phonemes. Along the advantage of a top-down approach, where complex tasks can be decomposed into atomic APs, this also enables a bottom-up approach whereby APs can be reused to construct different task sequences.

\section{PROPOSITION}

In [9] the authors proposed a solution to model the interactions between an intelligent mobility aid and their human operator. Navigational activities were modelled by decomposing them into primitive actions based on junctions and segments made available through a topological representation of the environment. The bottleneck in modelling ADLs with that approach was the need of generating a map prior to encoding the ADL activities. In this paper we present an alternative proposal in which activities are decomposed into a dictionary of APs based on intrinsic human motion behaviours, as perceived by the low level sensors fitted on the mobility device (e.g. "go straight". or "turn left" for the case of the ambulatory assistive device using in this work to demonstrate the proposed framework). This generic representation of APs appears to capture humancentered robot interactions more efficiently by allowing us to compute activity estimates without the need to resort to prior navigational maps, while still achieving as good inference accuracies (99.93\% vs $98.15 \%$ with mapping AP encodings).

The proposed probabilistic model is a Hierarchical Hidden Markov Model (HHMM), a stochastic framework operating 
at various levels of abstraction, hence naturally able to decompose complex ADLs into simpler APs. The building blocks that form the dictionary of APs act to describe the set of activities the user performs - with the aid of an instrumented walking device in this work. Given the multilevel decomposition of ADLs into APs, it will be shown how the HHMM framework can learn ADLs which are both static (activities performed at a single place, such as standing up) and navigational (moving to a specific location of interest, e.g. to kitchen) in nature.

To further validate the framework and demonstrate its effectiveness, data related to motion patterns and other compatible assistive behaviours was collected from a group of healthy volunteers while using a powered rollator walker, and results compared with standard discriminative and generative models.

\section{RELATED WORK}

There are significant challenges when modelling human behaviours in a deterministic manner, most notably the intrinsic uncertainty present when users perform an activity, and the inherent noise embedded in the sensor information gathered to provide cues about the behaviours. Researchers have therefore resorted to explore different stochastic/probabilistic modelling techniques capable of representing the possible uncertainties involved in ADLs. Probabilistic models such as Bayesian Network (BN) [13], Dynamic Bayesian Network (DBN) [14],Partially Observable Markov Decision Process(POMDPs) [15], [16], [17], Hidden Markov Models (HMM) [18] and Hierarchical Hidden Markov Models (HHMM) [19] have been proposed in the literature.

Hoey et. al. [17] for instance used a POMDP for computing policies and action to estimate and adapt to user states, such as awareness, responsiveness and overall dementia level. An HHM and Conditional Random Fields (CRFs) technique was utilised by Omar et. al. [20] to recognise activities of passive rollator walker user. The activities modelled were related to basic activities such as: stop/standing, walking forward, turn left, turn right, not touching the walker and transfers from sit to stand and vice versa. A Hierarchical Semi-Markov Model (HSMM) was proposed by Glover et. al. [3] to learn users' high level activities which were based on the topological location of the user and the most likely activity a user could perform at the respective location. The HSMM framework operated at three different levels: at the lowest level the metric motion is described by metric coordinates, at the mid-level the framework uses topological regions as its element and at the highest level the person's activities are divided into logically broader activities. Hirata et. al. [21] proposed a motion control algorithm to estimate user states of a passive walker user. The algorithm recognised three user states such as: walking, stopping and emergency falling. The user states were inferred based on the distance between the user and the walker and the velocity of the walker.

HHMMs have been proposed in several application areas. Nguyen et. al. [19] used an HHMM framework to model and recognise three human activities (e.g. having a meal) in a confined space, while tracking the user with two static cameras. The semantics embedded in the activities were tightly coupled to the locations where the relevant objects of interest - such as fridge, cupboard etc. - were located, not the actual interactions between user and objects. Grasping and object manipulation activities have also been studied with RGB-D cameras within the context of HHMM frameworks [22] in small 3D working envelopes. A framework based on an $\mathrm{H}-$ DBN was able to infer user's mode of transportation and desired destination [23] in an urban setting, and guidance cues where proposed when it was felt the user was deviating from his normal activities.

In user activity models related to robotic walker platforms (e.g. [20], [3], [21]), propositions have been mostly aimed at predicting either the fundamental user activities broadly aligned with what we categorise in our work as APs, or more abstract long term activities (as in the case of Glover et. al. [3]). In the approach suggested in this work, an HHMMbased model is used not only to infer APs, but also exploits these APs in a more sophisticated hierarchical structure to construct and identify overall activities of daily living (e.g. navigating to a location of interest) by probabilistically combining them into sequences of abstract human motion behaviours. The authors believe that such approach provides a natural framework to map continuous human-machine interaction behaviours, as those present when operating a typical walker frame, thus allowing more intelligent assistance with robotic mobility aids based on user intentions, ultimately the objective of this research.

\section{HIERARCHICAL HIDDEN MARKOV MODEL (HHMM)}

Hierarchical Hidden Markov Model (HHMM) generalises the standard Hidden Markov Models (HMM), by allowing hidden states to represent stochastic process themselves. HHMMs have become a popular tool for modelling real world data which has non-linear variations and hierarchical structure [24]. In HHMMs the state can either emit a single observation or string of observations. Single observations states are called "production state" whereas states that emit string of observations are called "abstract states" [25]. The high-level ("abstract states") emit a string of observations which are governed by sub-HMMs which are further recursively called till it emits a single ("production") state [25]. On execution of the sub-HHM, control is returned to wherever it was called from [25]. The hierarchical structure makes the HHMM capable of disintegrating a given problem at different levels of hierarchy thereby facilitating exploration (modelling long term activities) and exploitation (modelling action primitives) within the same framework. In the paradigm of modelling ADLs from action primitives (APs), the high level activities call a more refined string of low-level activities which in turn calls low level activities. This process continues till the most primitive activity state is executed. On termination of lower level activities, the parent behaviour may terminate or change state depending on its destination states. 


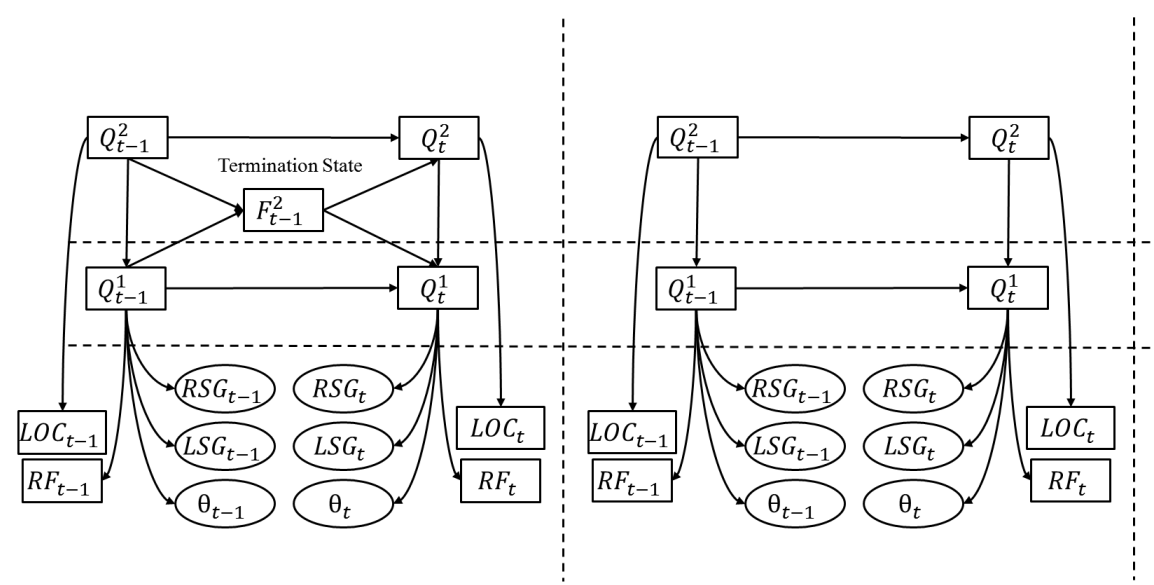

(a) HHMM model (b) Layered Dynamic Bayesian Network (DBN) model

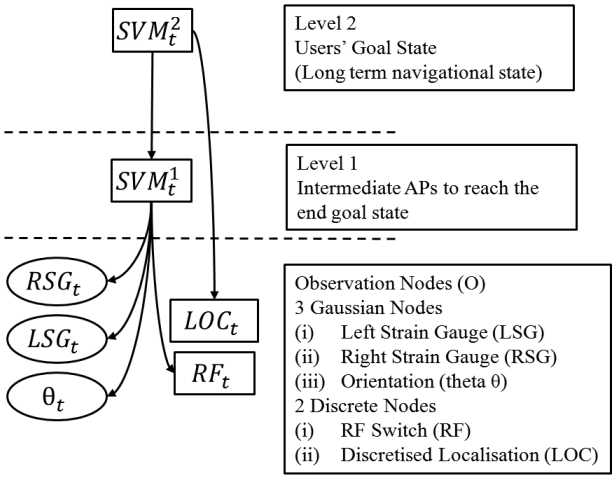

(c) Support Vector Machine (SVM) classifier

Fig. 1. (a) A 2-level HHMM model represented using a Hierarchical-DBN. The hierarchy of the framework demarcated by horizontal dashed lines. (b) 2-layer DBN Model in which layer 1 predicts the APs whereas layer 2 used the predicted APs and LOC information to infer long term tasks. (c) Discriminate SVM classifier for predicting APs and long term tasks.

A Hierarchical Dynamic Bayesian Network (H-DBN) representation of the HHMM framework is shown in Fig. 1(a). The H-DBN structure consists of three types of nodes, $Q_{t}^{d}, O_{t}, F_{t}^{d}$ where $d$ defines the hierarchical depth of the framework ( $d=2$ in our case). The edges connecting each nodes defines the dependencies between the states represented by each node.

Node $Q_{t}^{d}$ symbolises the hidden state at each level $d$ and time instance $t$. It is important to note that at a given time instance, hidden nodes at all the levels will be represented probabilistically by a state belief. The observation node $O_{t}$ provides information related to the user/environment to the hidden nodes of the framework. Based on the type of observation they can be modelled as discrete $P\left(Q_{t}^{d} \mid O_{t}\right)$ or mixture of Gaussian $(\mu, \Sigma)$ nodes. Lastly, node $F_{t}^{d}$ represents the terminating state of the sub-HMM so that the control can returned to the high level parent node/state.

Given the parameters $\left(Q_{t}^{d}, O_{t}, F_{t}^{d}\right)$, the H-DBN defines the joint probability distribution over the set of variables that represents the evolution of the stochastic process over time. The state distribution are defined in the form of prior probabilities $\left(\pi_{1}^{d}\right)$, transition probabilities $\left(A(i, j)_{t}^{d}\right)$, observation probabilities $\left(O_{t}\right)$ and termination probabilities $(A(i, e n d))$.

\section{A. Prior Probabilities}

The prior probabilities provide information related to the most likely initial user state. The probabilities at level 1 and 2 are defined as

$$
\begin{aligned}
& P\left(Q_{1}^{2}\right)=\pi^{2}(j) \\
& P\left(Q_{1}^{1}\right)=\pi_{k}^{1}(j),
\end{aligned}
$$

where $\pi^{2}$ represent the initial probabilities at level 2 and $\pi_{k}^{1}$ at level 1 , provided level 2 is in state $k$.

\section{B. Transition Probabilities}

The transition probabilities of an HHMM framework represent the most likely state of the system, represented using a conditional probability table or density (CPT). The probability distribution at level 2 is conditioned on the previous state at the same level, and the termination state at time $t-1$. The Probabilities at level 2 are defined as

$$
P\left(Q_{t}^{2}=j \mid Q_{t-1}^{2}=i, F_{t-1}^{2}=f\right)= \begin{cases}A^{2}(i, j) & \text { if } F_{t-1}^{2}=0 \\ \pi^{2}(j) & \text { if } F_{t-1}^{2}=1\end{cases}
$$

Similarly, the conditional probabilities at level 1 (Fig. 1(a)) at time $t$ has its dependencies on the state at level 2 at time $t$, the previous state at level 1 , and the termination state at time $t-1$. The CPTs at the intermediate level are defined by

$$
P\left(Q_{t}^{1}=j \mid Q_{t-1}^{1}=i, F_{t-1}^{2}=f, Q_{t}^{2}=k\right)= \begin{cases}A_{k}^{1}(i, j) & \text { if } F_{t-1}^{2}=0 \\ \pi_{k}^{1}(j) & \text { if } F_{t-1}^{2}=1 .\end{cases}
$$

In (2), $A^{2}$ represents the transition probabilities from state $i$ to $j$. Similarly, in (3), the transition probability at level 1 given level 2 is in state $k$ is captured in $A_{k}^{1}$.

\section{Termination Probabilities}

The termination state $F$ has its dependencies on the hidden states at level 1 and level 2 at time step $t$. CPTs of the termination state is defined by

$$
P\left(F_{t}^{2}=1 \mid Q_{t}^{2}=k, Q_{t}^{1}=i\right)=A_{k}^{2}(i, \text { end }) .
$$

\section{Observation Probabilities}

The observation probabilities are conditioned on the hidden state provided a specific observation is observed. The observation probabilities for discrete and observed nodes is given by

$$
\begin{aligned}
& P\left(O_{t} \mid Q_{t}^{1}=i\right)=N\left(\mu_{i}, \Sigma_{i}\right) \\
& P\left(O_{t} \mid Q_{t}^{1}=i\right)=C(i) .
\end{aligned}
$$

\section{E. Learning and Inference}

The HHMM framework can be learned using both supervised and unsupervised learning techniques. For unsupervised learning, Expectation Maximisation (EM) [26] is probably the most widely used learning technique. It is a general iterative technique for performing maximum likelihood estimation of parameters which consists of missing data. It is generally rather difficult to obtain labelled data when 
TABLE I

USERS' EVERYDAY ACTIVITIES

\begin{tabular}{lcl}
\hline User Task & Abbrev. & Description \\
\hline Recall Walker & RW & Recalling walker to use it \\
Walker Go Away & WGA & Instruction the walker to go way so that it is not an hindrance \\
Stand Up & SU & Stand up using the support of the walker \\
Sit Down & SD & Sit down using the support of the walker \\
to Bedroom & BED & Navigating to the bedroom \\
to Kitchen & KIT & Navigating to the kitchen \\
to Bathroom & BAT & Navigating to the bathroom \\
to Laundry & LAU & Navigating to the laundry \\
to Medical & MED & Navigating to the medical \\
Navigating in Room & GAN & Navigating locally in a room \\
\hline
\end{tabular}

dealing with realistic scenarios, thus an unsupervised mode of learning is preferable. In our work the model was therefore learnt with EM, and a maximum likelihood estimator was then employed to infer the activities.

\section{PROBLEM SPECIFIC HHMM FRAMEWORK}

Everyday user activities in our experimental set-up were organised as per those in Table I. The tasks are a combination of static activities generally associated to being performed at a single location (e.g Stand Up), and goal-oriented navigational tasks (e.g Going to Kitchen) which involve visiting different location of interest. Static activities being already in their most primitive state cannot be decomposed further, hence such ADLs are also regarded as independent APs in themselves. On the other hand, goal-oriented activities need to be further decomposed into more basic navigational components, which in this work are proposed to be in the form of relative human motion behaviours (e.g Turn Left) to avoid the bottleneck of encoding absolute navigational information from a map, and the increased AP dimensionality that comes with it (10 vs 17 with the map-based AP strategy). The overall number of APs in which the tasks have been clustered are listed in Table II.

A typical scenario could be illustrated by considering for instance the activities involved in going to the kitchen from the bedroom (shown in light blue in Fig. 2(b)). The sequence of tasks can be decomposed as "Recall Walker $\Rightarrow$ Stand $U p \Rightarrow$ Navigate in Room (until out onto the corridor) $\Rightarrow$ To Kitchen [Go Straight Southwards $\Rightarrow$ Turn Right $\Rightarrow$ Go Straight Westwards (until entering kitchen)] $\Rightarrow$ Navigate in Room $\Rightarrow$ Sit Down $\Rightarrow$ Walker Go Away".

The HHMM model used to evaluate our proposition is shown in Fig. 1(a). In this case, user activities are layered in two abstraction levels wherein APs are predicted at the intermediate level (level 1), and ADLs are inferred at the higher level. As in our previous work, the observation nodes of the framework are a combination of both the sensors installed on the walker, and location information attained from a localisation module:

- The readings from the physical sensors of the walker (LSG,RSG) and orientation ( $\theta$ ) information are conditioned as Gaussian nodes.

- Two discrete nodes, a location of interest (LOC) obtained from the $(x, y)$ location of the user and walker, and an RF node corresponding to an RF switch used to recall the walker or to indicate it to go away.
TABLE II

ACTION PRIMITIVES

\begin{tabular}{lcl}
\hline Action Primitive & Abbrev. & Description \\
\hline Recall Walker & RW & Recalling walker to use it \\
Walker Go Away & WGA & Instruction the walker to go way so that it is not an hindrance \\
Stand Up & SU & Stand up using the support of the walker \\
Sit Down & SD & Sit down using the support of the walker \\
Go Straight Northwards & GSN & Going straight with orientation towards north \\
Go Straight Westwards & GSW & Going straight with orientation towards west \\
Go Straight Eastwards & GSE & Going straight with orientation towards east \\
Go Straight Southwards & GSS & Going straight with orientation towards south \\
Turn Left & TL & Turning left \\
Turn Right & TR & Turning right \\
\hline
\end{tabular}

Our office space was divided into different location of interest so as to resemble a typical home setting. User would then visit some of these many locations while performing their different everyday activities with the aid of the assistive walker, as described in the next Section.

\section{EXPERIMENTAL RESULTS \& DISCUSSION}

Off-line testing of the HHMM framework was done using real time data collected from a set of users while they performed different activities using an instrumented walker platform

\section{A. Power Walker for Data Acquisition}

The power walker used for data collection is a modified version of a commercially available walking frame with four wheels shown in Fig 2(a). Two 24Volts (DC) reversible gearhead motors, along with incremental optical encoders were fitted to the rear wheels of the walker (front wheels were passive). Two set of strain gauges (SGs) bridges were mounted on each handle bar. The differential force measured on the vertical axis on each handle-bar was used as an indicative measure of the users' intention to start an activity (e.g. user trying to stand up). The platform was also instrumented with a radio switch, used to instruct the walker to go to a parking position, or come back to the last location where the user sent the walker away. This feature is beneficial in places where the user performs activities that do not required walker support (e.g. while sitting watching TV). A microcontroller at the lower level was used to communicate with the sensor hardware and a high performance computer was also installed on the walker for data processing.

Given the nature of the work, and the sensitivity of undertaking trials with aged/frail subjects, healthy volunteers (25-31 years of age) were asked to participate in a set of experiments. The volunteers were briefed on the working of the platform and given sufficient time to understand the functionality and practice using the walker. During the experiments, the powered walker was configured to simply provide steering assistance based on data sensed from the handle gauges, i.e. no further active navigational support was provided.

A key criteria to provide active assistance to walker users in their everyday activities is of course to acquire knowledge about their activity patterns. Typically, walker users have a known set of target locations such as bathroom, kitchen, living room etc, that they visit while performing different daily activities. Identifying locations of interest is not straightforward and there is no general method to 


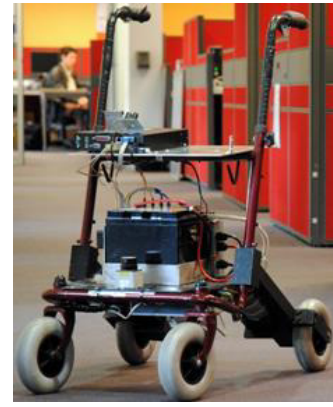

(a) Intrumented rollator walker

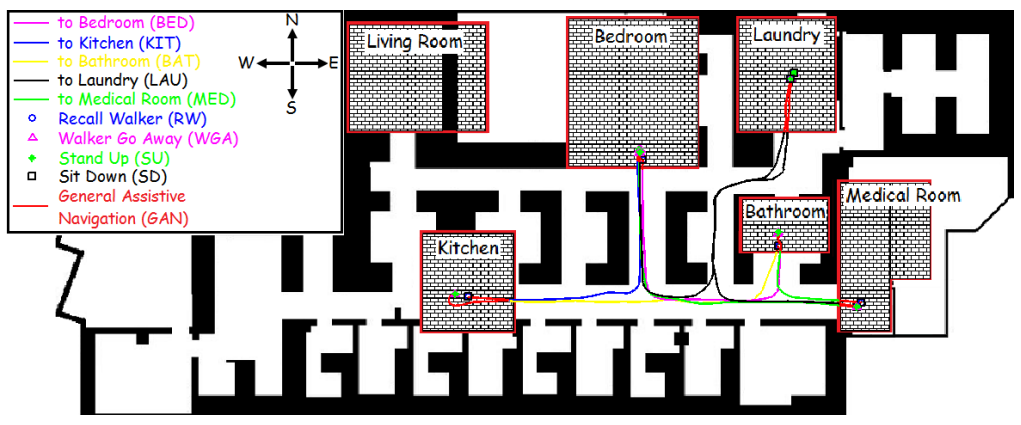

(b) Set of ADLs performed by one of the users in the testing environment

Fig. 2. (a) The instrumented power rollator walker used for data collection. (b) Top view of the testing environment, divided into different typical locations of interest in a home setting, where walker user ADLs are superimposed in different colour depending on the routine.

achieve this, as it is a user and environment specific problem. These patterns are normally defined by occupational therapist while users' activities are monitored under clinical tests conditions [27]. In this work, however, we predefined the activities encountered by a walker user. 5 different locations of interest generally reflective of a home environment were set and data from the sensors collected while user visited those as they wished. An example of a trajectory plot from one of the users is shown in Fig. 2(b). Data was logged at a sampling rate of $0.5 \mathrm{~Hz}$ using the Player/Stage open source toolbox [28].

\section{B. Methodology}

The proposed HHMM framework to learn and infer user activities was evaluated using the BNT toolbox [25]. Collected data was divided into two equal sets for training and testing purposes. To further cross validate the inference accuracy the data was manually labelled. The ADLs were inferred at a higher level (level 2), with an accuracy of 99\% whereas the APs were inferred with an accuracy of $88 \%$ at the intermediate level using the HHMM framework (see Tables III and IV respectively). Overall accuracy is only marginally better than in our previous work (98.15\%), yet the network was able to achieve this high inference of behavioural accuracy without the need to resort to absolute topological information from the map in their APs, a significantly more computationally efficient strategy in generalising the hierarchical network to broader domains.

\section{Discussion}

To further validate the inference capabilities of our proposed scheme, we also compared the results against two of the main approaches used in human activity recognition. DBN is probably the most common and better suited, yet a flat network would be an unfair comparison, and for this work the layered DBN shown in Fig. 1(b) was used. In this model, layer 1 infers the APs, whereas layer 2 uses the localisation information and the APs inferred by layer 1 to predict the long term tasks. As seen in Tables III and IV, the inference accuracies of the APs were necessarily very similar to those attained with the HHMM framework, yet drastically reduced for ADLs. This further reinforces the capability of the hierarchical framework, which exploits the spatio-temporal nature of ADLs. The results of an HHMM framework were also compared with a common discriminative classifier such as SVM. Once again, to make a fair comparison, two separate SVMs were used to predict the APs and ADLs, as depicted by Fig. 1(c). The APs are predicted by $S V M^{1}$, which were then used as inputs to the $S V M^{2}$ classifier, along with localisation information, to infer ADLs. Since SVMs learning is supervised, classification accuracy of APs was necessarily higher, in the order of $96 \%$, as the atomic classes are more easily differentiable, yet the temporal constraints in the ADL classification cannot be so easily encapsulated by maximum margin approaches such as SVM, and accuracies around $76 \%$ were attained.

\section{CONCLUSION \& FUTURE WORK}

Robotic systems are bound to acquire more sophisticated assistive capabilities if they are to operate in unstructured, dynamic, human-centred environments, responsive to the needs of interacting with humans. Within that context, awareness of human intentions play a key role to be able to apply any practical assistive action where a user interacts with a robot to carry out their regular daily activities, be that navigational, manipulative, communicative, or others. This work postulates a cognitive system that exploits hierarchical structures within temporal models to infer user behaviours while carrying out a set of interacting activities. A specific example of ADLs framed within the context of an ambulatory robot aimed at providing assistance to users with mobility deficiencies has been studied. The alternative proposition in this work of encoding intrinsic human motion behaviours has been shown to outperform an earlier suggestion that used topological information to form the chain of APs, while at the same time being computationally more effective as the dimensionality of the APs is also reduced. Real data collected from (able) users of an intelligent robotic rollator walker has been used to evaluate the proposed work. The framework has been shown capable of predicting APs and recognise long term user intentions generated by combining APs in varying sequential orders. The proposed HHMM model inferred APs with an accuracy of $88 \%$, but rather significantly ADLs were predicted with an accuracy of $99 \%$ (marginally better than the $98.15 \%$ achieved with mapping AP encodings). For validation purposes, the HHMM model was also compared 
TABLE III

ACCURACY OF ADLS INFERRED BY GENERATIVE AND DISCRIMINATIVE MODELS (\%)

\begin{tabular}{|c||c|c|c|c|c|c|c|c|c|c|c|}
\hline Model / $A D L$ & BED & KIT & BAT & MED & LAU & RW & WGA & SU & SD & GAN & Overall \\
\hline \hline HHMM & 100.00 & 100.00 & 100.00 & 100.00 & 100.00 & 100.00 & 100.00 & 100.00 & 97.22 & 100.00 & $\mathbf{9 9 . 9 3}$ \\
\hline DBN & 16.00 & 56.00 & 89.00 & 57.00 & 62.00 & 100.00 & 100.00 & 100.00 & 100.00 & 100.00 & $\mathbf{6 7 . 5 8}$ \\
\hline SVM & 41.69 & 0.00 & 59.76 & 93.71 & 93.81 & 100.00 & 99.09 & 98.31 & 100.00 & 100.00 & $\mathbf{7 6 . 4 7}$ \\
\hline
\end{tabular}

TABLE IV

APS INFERENCE ACCURACY BY GENERATIVE AND DISCRIMINATIVE MODELS (\%)

\begin{tabular}{|c||c|c|c|c|c|c|c|c|c|c|c|}
\hline Model / AP & RW & WGA & SU & SD & TL & TR & GSS & GSW & GSN & GSE & Overall \\
\hline \hline HHMM & 100.00 & 100.00 & 100.00 & 97.22 & 77.01 & 51.93 & 95.90 & 92.49 & 90.92 & 80.70 & $\mathbf{8 8 . 0 5}$ \\
\hline DBN & 100.00 & 100.00 & 100.00 & 100.00 & 52.38 & 35.21 & 96.04 & 94.40 & 92.05 & 86.59 & $\mathbf{8 7 . 7 9}$ \\
\hline SVM & 100.00 & 99.09 & 98.31 & 100.00 & 82.89 & 64.38 & 98.67 & 100.00 & 98.40 & 97.66 & $\mathbf{9 6 . 0 9}$ \\
\hline
\end{tabular}

with two common comparable techniques, a layered DBN and two sequential SVMs. All three techniques predicted APs with reasonable accuracies, yet the inference of ADLs reduced substantially for the layered DBN and 2 stage SVM models, which substantiates the relevant role of temporal relations in predicting complex ADLs. This work is currently focused on incorporating an online learning algorithm within the HHMM framework, such as online-EM, so that the model can adapt to changes in user behaviours, and/or daily patterns, both of which are likely to occur with time.

\section{REFERENCES}

[1] S. Dubowsky, F. Génot, S. Godding, H. Kozono, A. Skwersky, H. Yu, and L. S. Yu, "PAMM - A robotic aid to the elderly for mobility assistance and monitoring: A helping-hand for the elderly," in IEEE International Conference on Robotics and Automation, 2000, pp. 570 $-576$.

[2] T. Carlson and Y. Demiris, "Increasing robotic wheelchair safety with collaborative control: Evidence from secondary task experiments," in IEEE International Conference on Robotics and Automation, 2010, pp. $5582-5587$.

[3] J. Glover, S. Thrun, and J. T. Matthews, "Learning user models of mobility-related activities through instrumented walking aids," in IEEE International Conference on Robotics and Automation, 2004, pp. 3306 -3312 .

[4] G. Wasson, P. Sheth, C. Huang, and M. Alwan, "Intelligent mobility aids for the elderly," in Eldercare Technology for Clinical Practitioners, M. Alwan and R. A. Felder, Eds. Humana Press, 2008, pp. 53 $-76$.

[5] S. Srinivasa, D. Ferguson, M. Vandeweghe, R. Diankov, D. Berenson, C. Helfrich, and H. Strasdat, "The robotic busboy: Steps towards developing a mobile robotic home assistant," in International Conference on Intelligent Autonomous Systems, 2008, pp. 129-136.

[6] United Nations, "United nations 2006 world populations prospects: The 2006 revision," United Nations, New York, Tech. Rep., 2006.

[7] M. M. Martins, C. P. Santos, A. Frizera-Neto, and R. Ceres, "Assistive mobility devices focusing on smart walkers: Classification and review," Robotics and Autonomous Systems, vol. 60, no. 4, pp. 548-562, 2012.

[8] A. Officer and A. Posarac, World Report on Disability. World Health Organisation, 2011.

[9] M. Patel, J. V. Miró, and G. Dissanayake, "A hierarchical hidden markov model to support activities of daily living with an assistive robotic walker," in 4th IEEE/RAS EMBS International Conference on Biomedical Robotics and Biomechatronics, 2012, pp. 1071 - 1076.

[10] V. Krüger, D. Herzog, S. Baby, A. Ude, and D. Kragic, "Learning actions from observations," IEEE Robotics \& Automatation Magazine, vol. 17 , no. 2 , pp. $30-43,2010$.

[11] D. Kulic, D. Kragic, and V. Krüger, "Learning action primitives," in Visual Analysis of Humans, 2011, pp. 333 - 353.

[12] G. Rizzolatti, L. Foggassi, and V. Gallese, "Neurophysiological mechanisms underlying the understanding and imitation of action," Nature Reviews Neuroscience, vol. 2, pp. $661-670,2001$.
[13] J. H. Hong, Y. S. Song, and S. B. Cho, "A hierarchical bayesian network for mixed-initiative human-robot interaction," in IEEE International Conference on Robotics and Automation, 2005, pp. 3808 3813.

[14] K. A. Tahboub, "Intelligent human-machine interaction based on dynamic bayesian networks probabilistic intention recognition," Journal of Intelligent and Robotic Systems, vol. 45, no. 1, pp. 31 - 52, 2006.

[15] J. V. Miró, V. Osswald, M. Patel, and G. Dissanayake, "Robotic assistance with attitude: A mobility agent for motor function rehabilitation and ambulation support," in IEEE International Conference on Rehabilitation Robotics, 2009, pp. 529 - 534.

[16] T. Taha, J. V. Miró, and G. Dissanayake, "Pomdp-based long-term user intention prediction for wheelchair navigation," in IEEE International Conference on Robotics and Automation, 2008, pp. 3920 - 3925.

[17] J. Hoey, A. V. Bertoldi, P. Poupart, and A. Mihailidis, "Assisitng persons with dementia during handwashing using a partially observable markov decision process," in the International Conference on Vision Systems, March 2007.

[18] C. Zhu, Q. Cheng, and W. Sheng, "Human intention recognition in smart assisted living systems using a hierarchical hidden markov model," in IEEE International Conference on Automation Science and Engineering, 2008, pp. $253-258$.

[19] N. Nguyen, D. Phung, S. Venkatesh, and H. Bui, "Learning and detecting activities from movement trajectories using the hierarchical hidden markov model," in IEEE Computer Society Conference on Computer Vision and Pattern Recognition, vol. 2, 2005, pp. 955 960.

[20] F. Omar, M. Sinn, J. Truszkowski, P. Poupart, J. Tung, and A. Caine, "Comparative analysis of probabilistic models for activity recognition with an instrumented walker," in the 26th Conference on Uncertainty in Artificial Intelligence, 2010, pp. 1 - 9.

[21] Y. Hirata, A. Muraki, and K. Kosuge, "Motion control of intelligent passive-type walker for fall-prevention function based on estimation of user state," in IEEE International Conference on Robotics and Automation, 2006, pp. 3498 - 3503.

[22] M. Patel, C. H. Ek, N. Kyriazis, A. Argyros, J. V. Miro, and D. Kragic, "Language for learning complex human-object interactions," in IEEE International Conference on Robotics and Automation, 2013, pp. 4982 $-4987$.

[23] L. Liao, "Location-based activity recognition," Ph.D. dissertation, University of Washington, 2006.

[24] S. Fine, Y. Singer, and N. Tishby, "The hierarchical hidden markov model: Analysis and applications," Machine Learning, vol. 32, pp. 41 $-62,1998$.

[25] K. P. Murphy, "Dynamic bayesian networks: Representation, inference and learning," Ph.D. dissertation, University of Califronia, Berkeley, 2002.

[26] A. P. Dempster, N. M. Laird, and D. B. Rubin, "Maximum likelihood from incomplete data via the em algorithm," Journal of Royal Statistical Society, Series B, vol. 39, no. 1, pp. 1-38, 1977.

[27] O. T. Practice, "Occupational therapy practice framework : Domain \& process (2nd edition)," The American Journal of Occupational Therapy, vol. 62, no. 6, pp. 625-683, 2008.

[28] B. Gerkey, R. Vaughan, and A. Howard, "The player/stage project: Tools for multi-robot and distributed sensor systems," in The 11th IEEE International Conference on Advanced Robotics, 2003, pp. 317 -323 . 